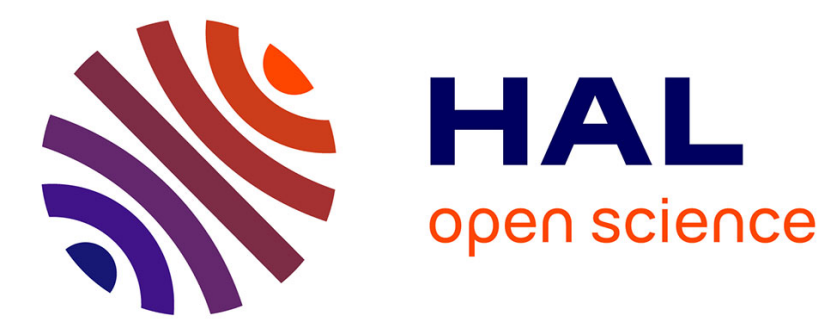

\title{
La production incunable vénitienne et le milieu du livre. Une approche quantitative et comparative
}

Catherine Kikuchi

\section{To cite this version:}

Catherine Kikuchi. La production incunable vénitienne et le milieu du livre. Une approche quantitative et comparative. Gazette du Livre Médiéval, 2014, 61, pp.36-54. hal-01526733

\section{HAL Id: hal-01526733 \\ https://hal.science/hal-01526733}

Submitted on 23 May 2017

HAL is a multi-disciplinary open access archive for the deposit and dissemination of scientific research documents, whether they are published or not. The documents may come from teaching and research institutions in France or abroad, or from public or private research centers.
L'archive ouverte pluridisciplinaire HAL, est destinée au dépôt et à la diffusion de documents scientifiques de niveau recherche, publiés ou non, émanant des établissements d'enseignement et de recherche français ou étrangers, des laboratoires publics ou privés. 
Catherine Kikuchi

\title{
LA PRODUCTION INCUNABLE VENITIENNE ET LE MILIEU DU LIVRE
}

\author{
UNE APPROCHE QUANTITATIVE ET COMPARATIVE
}

Dès 1478, neuf ans après l'introduction de l'imprimerie dans la lagune, Venise devient la première ville productrice d'imprimés, place qu'elle gardera pendant la quasi-totalité de la période de l'incunable1. Les presses de la cité dépassent alors la production de Rome, qui rentre à ce moment-là en crise. Ce n'est qu'à partir de 1490 que Venise trouve une concurrente qui puisse se comparer à elle, Paris, qui finit le siècle avec une production supérieure d'une quinzaine d'éditions à la production vénitienne. Venise inonde le marché européen. On retrouve ses éditions dans toute l'Italie et sur tout le continent : entre autres, dans les pays germaniques, grâce à un réseau commercial bien développé vers le Nord de l'Europe; en France, notamment grâce à ses relations commerciales avec Lyon; ou encore dans la Péninsule ibérique, où les Vénitiens envoient des libraires les représentant.

Cette production a déjà été beaucoup étudiée de différents points de vue. Les imprimeurs les plus connus ont fait l'objet de monographies. Martin Lowry, en particulier, a renouvelé le genre en étudiant Nicolas Jenson et Alde Manuce sous l'angle de leurs relations sociales et économiques2. Depuis quelque temps, des auteurs tels que Cristina Dondi s'intéressent à la distribution des livres vénitiens et à leur commercialisation, notamment à travers un ambitieux programme de recherche actuellement en cours3. Des

\footnotetext{
${ }^{1}$ Nous utilisons ici par commodité le terme «incunable » et la borne traditionnelle de 1500. Cette date est surtout utilisée dans cet article en raison des bases de données disponibles. Cependant, il est certain que s'arrêter en 1500 n'a que peu de sens du point de vue de l'histoire du livre, comme nous le verrons d'ailleurs.

2 M. Lowry, Le Monde d'Alde Manuce. Imprimeurs, hommes d'affaires et intellectuels dans la Venise de la Renaissance, Évreux, 1989 (1979) ; M. LOWRY, "Nicholas Jenson and the Rise of Venetian Publishing and Renaissance Europe", Oxford, 1991.

${ }^{3}$ Voir C. DoNDI, "The $15^{\text {th }}$-Century Booktrade : An Evidence-based Assessment and Visualization of the Distribution, Sale and Reception of Printed Books in the Renaissance", dans $G L M$ n $^{\circ} 60$ (année 2013), p. 83-101.
} 
2 -Gazette du livre médiéval, $\mathrm{n}^{\circ} \#$ \#, 20\#, fasc. \#.

travaux en histoire moderne prolongent ces préoccupations, notamment ceux de Mario Infelise ou Angela Nuovo4.

L'approche quantitative a été relativement peu utilisée pour étudier la production libraire de Venise, si ce n'est par ceux qui en sont les initiateurs. Ainsi, Dominique Coq et Ezio Ornato ont pu mettre en lumière la spécialisation de Venise dans le domaine du livre juridique5. Une étude d'ensemble a été tentée par Leonard Gerulaitis, qui cependant ne s'appuie pas sur une base de données exhaustive 6 ; par ailleurs, son analyse nous semble à revoir sur certains points. Nous tenterons donc ici de dresser les grandes lignes de la production vénitienne, en nous inspirant notamment du travail de Chiara Ruzzier, Xavier Hermand et Ezio Ornato sur la production des anciens Pays-Bas7. Ici, comme dans cet ouvrage, il s'agira de mettre au jour les stratégies éditoriales des imprimeurs et les modèles qui s'en dégagent. Par «stratégie éditoriale », les auteurs entendent «l'ensemble des choix de toutes sortes opérés par un imprimeur dans le but d'écouler le plus rapidement possible sur le marché la totalité de sa production $\gg 8$ : c'est en ce sens que nous emploierons la même expression. Cela dit, cet article ne prétend pas épuiser une question aussi vaste que celle de la production vénitienne, mais seulement identifier des tendances qui demanderont par la suite à être précisées et approfondies.

Pour mener à bien ce travail, nous avons utilisé une base de données où, aux données de l'ISTC et du Gesamtkatalog der Wiegendrucke, ont été ajoutés de nouveaux champs, notamment un essai de classification textuelle et la date de mort des auteurs des textes publiés9. La démarche consiste ensuite de prendre des indicateurs un par un pour étudier l'évolution de la production vénitienne, tout en prenant garde aux erreurs et imprécisions

${ }^{4}$ On notera en particulier l'ouvrage de synthèse d'A. NuOvo, Il Commercio librario nell'Italia del Rinascimento, Milan, 1998, récemment réédité et augmenté dans une version anglaise, The Book Trade in the Italian Renaissance, Leiden, 2013.

${ }^{5}$ D. Coq et E. Ornato, «La Production et le marché de l'incunable. Le cas des livres juridiques », dans La Face cachée du livre médiéval. L'histoire du livre vue par Ezio Ornato, ses amis, ses collègues, Rome, 1997, p. 227-244.

${ }^{6}$ L. V. Gerulaitis, Printing and Publishing in Fifteenth-Century Venice, Chicago, American Library Association, 1976.

${ }^{7}$ C. RuZzIER, X. Hermand, E. Ornato, Les Stratégies éditoriales à l'époque de l'incunable : le cas des anciens Pays-Bas, Turnhout, 2012.

${ }^{8}$ Ibid., p. 10.

${ }^{9}$ La constitution de cette base de données par Ezio Ornato et Claire Priol, est décrite Ibid., p. 15 et suiv. Je remercie ici très sincèrement Ezio Ornato qui a bien voulu me la laisser exploiter. Cet article est largement le fruit de nos discussions à ce sujet. 
inévitables dans une enquête de ce genre. En particulier, si le taux d'éditions non datées à Venise est nettement inférieur à celui d'autres villes, il représente malgré tout $25 \%$ de la production, ce qui pourrait introduire un biais dans l'analyse chronologique ; cependant, la très grande majorité de ces éditions ont reçu une date approximative fondée sur la critique, qui est mentionnée par l'ISTC et qui a été prise en compte. D'un autre côté, le problème des éditions perdues est moins aigu à Venise que dans d'autres villes, dans la mesure où l'essentiel de la production est constitué par des éditions dont le taux de survie est suffisamment élevé pour qu'au moins un exemplaire soit parvenu jusqu'à nous.

Il faut également choisir les points sur lesquels la production vénitienne devra être mise en perspective avec celle d'autres centres de production. Cette comparaison s'impose afin de ne pas considérer la production vénitienne comme une entité indépendante de celle des autres villes d'imprimerie : au contraire, il s'agit de replacer Venise dans un contexte de production et de diffusion qui devient rapidement européen. Les points de comparaison devront varier en fonction des aspects étudiés. Même si cette étude ne s'inscrit pas dans l'axe de l'« histoire comparée » à proprement parler, il s'agit d'analyser les presses vénitiennes sans jamais oublier les relations de concurrence avec d'autres centres, plus ou moins proches.

Enfin, au-delà de l'aspect quantitatif et comparatif de notre étude, nous voulons mettre en rapport ces premiers résultats avec une étude sociale et économique du milieu du livre vénitien, étant entendu que la production du livre ne peut être détachée des acteurs qui y participent. Nous pensons que l'étude des stratégies éditoriales des imprimeurs permet d'éclairer leurs interrelations économiques et sociales et ainsi de mieux comprendre le développement de l'imprimerie dans ses premiers temps.

\section{LES TROIS TEMPS DE LA PRODUCTION VENITIENNE}

Afin d'obtenir une vue d'ensemble de la production vénitienne entre 1469 , date du premier livre imprimé dans la cité, et 1500, limite conventionnelle de la période de l'incunable, nous avons utilisé des critères concernant aussi bien le contenu des ouvrages imprimés que leur matérialité. Nous avons ainsi relevé le format, la langue, le type de texte des ouvrages publiés, la date de mort des auteurs. Nous avons également cherché à croiser ces indicateurs pour éviter les biais éventuels engendrés par l'ambiguïté et l'inévitable dose d'arbitraire pouvant affecter certaines classifications, notamment pour ce qui est de la typologie textuelle10.

\footnotetext{
${ }^{10}$ Celle-ci est d'ailleurs en cours de révision, en particulier en ce qui concerne les catégories «littérature » et «théologie» dont la définition et la délimitation soulèvent un certain nombre de difficultés.
} 
Par ailleurs, les quelques éditions dont la date est totalement inconnue ne sont pas prises en compte ici. Afin de lisser certains pics locaux dus à l'arrondissement des datations au jugé, nous avons également choisi d'étudier la production par tranches de trois ans. Cela nous permet d'étudier des périodes comparables en termes de nombre d'éditions, moins soumises aux variations purement conjoncturelles de la production annuelle. Suivant cette méthodologie, nous avons pu dégager trois grandes périodes de la production libraire vénitienne.

TABLEAU 1. - Nombre d'éditions par tranches triennales

\begin{tabular}{|l|r|}
\hline $1469-1471$ & 98 \\
\hline $1472-1474$ & 166 \\
\hline $1475-1477$ & 200 \\
\hline $1478-1480$ & 222 \\
\hline $1481-1483$ & 289 \\
\hline $1484-1486$ & 282 \\
\hline $1487-1489$ & 302 \\
\hline $1490-1492$ & 453 \\
\hline $1493-1495$ & 508 \\
\hline $1496-1498$ & 451 \\
\hline $1499-1500$ & 494 \\
\hline
\end{tabular}

Une première phase s'étend de 1469 au deuxième tiers de la décennie 1470. Il s'agit d'une phase d'ajustements importants et de grandes fluctuations, surtout dans les premières années. Le contenu publié fait l'objet d'importants changements : ainsi, la production profane, qui atteint $85 \%$ au tout début, baisse de façon significative pour atteindre un point d'équilibre vers 1475-1477 ; à partir de ce moment, ces œuvres représentent entre 60 et $70 \%$ de la production totale (graphique 1). On peut également observer des fluctuations dans le format des éditions, mais l'in-folio finit par l'emporter durablement vers 1475 (graphique 2). Les évolutions sont moins accentués plus fines en ce qui concerne la langue du texte : dès les trois premières années d'activité typographique, le latin représente $86,7 \%$ des éditions vénitiennes; cette part descend légèrement, mais se maintient à environ $80 \%$ de la production pendant toute la période, avec quelques légères variations (graphique 3).

Si l'on considère les principales catégories textuelles (graphique 4), des changements sont observables jusque 1475 voire 1478, moment à partir duquel l'imprimerie vénitienne se concentre sur le droit et la littérature. À partir de ce moment, les deux domaines combinés regroupent entre 40 et $60 \%$ de la production de la ville, chacun représentant une part comparable dans la production totale. Des évolutions sont également sensibles en ce qui 
concerne les auteurs publiés: les auteurs de l'Antiquité, qui avaient constitué un marché juteux pour les toutes premières éditions vénitiennes, perdent progressivement leur suprématie pour ne plus représenter que $17,6 \%$ de la production en 1478-1481 (graphique 5); on observe inversement une hausse de la production d'auteurs des XI $\mathrm{C}^{\mathrm{e}}-\mathrm{XIII} \mathrm{e}^{\mathrm{e}}$ siècles ; ces éditions sont essentiellement destinées à un public universitaire : six éditions de Thomas d'Aquin11, deux de Bonaventure12, ou encore une édition de Pierre Lombard 13.

Ces changements illustrent bien l'orientation que prend alors l'imprimerie vénitienne. À la fin de cette première période, se dessine progressivement une production profane, en latin et dans de grands formats, concernant principalement le droit, les auteurs classiques et, en général, les ouvrages savants

À partir de la fin des années 1470, les tendances se manifestent sans équivoque et les imprimeurs vénitiens ont mis en place une stratégie éditoriale cohérente et stable. La décennie suivante confirme les évolutions que nous avons décrites précédemment. Si l'on regarde le choix des auteurs publiés, les grandes tendances sont déjà en place vers 1478 et perdurent ensuite ; il en va de même pour la répartition entre la production sacrée et profane, ou pour le format des éditions. Cependant, la progression des auteurs $d u X V^{e}$ siècle et des auteurs contemporains se fait plus prudente, après une croissance rapide dans les premières années, et met plus de temps à se stabiliser (graphique 5).

Malgré ces modifications et ces réajustements, il semble bien qu'à la fin des années 1470, la production vénitienne ait trouvé un certain point d'équilibre. Il s'agit d'une production destinée à un public savant, qui se spécialise en particulier dans le domaine juridique. Ce sont des éditions nécessitant des investissements importants, avec en particulier des sommes de grands formats. La production littéraire, les auteurs antiques notamment, continue de constituer un axe important, mais complémentaire de la production d'ouvrages de droit, qui culmine à la fin des années 1480 (graphique 4). Par ailleurs, la production de textes antiques change de fonction : on passe peu à peu des éditions destinées à un public aisé mais non «professionnel», remplaçant les élégants manuscrits d'auteurs classiques que l'on copiait auparavant sur parchemin en écriture

\footnotetext{
${ }^{11}$ La Somme théologique (ig00163000, it00198000 et it00204000) en trois volumes et le commentaire du quatrième livre des Sentences de Pierre Lombard (it00169000) notamment.

12 Le commentaire sur le second livre des Sentences de Pierre Lombard (ib00873000) et les Méditations sur la vie du Christ (ib00915500).

${ }^{13}$ Les Sentences (ip00480000).
} 
6 -Gazette du livre médiéval, $\mathrm{n}^{\circ} \#$ \#, 20\#, fasc. \#.

humanistique, à des éditions commentées dont l'usage était essentiellement scolaire14.

De nouveaux changements d'orientation sont notables durant les années 1490. Si l'on observe l'évolution de la part des auteurs en fonction de leur date de mort (graphique 5), la répartition est comparable à celle de la période précédente. Si l'on regarde par contre la répartition par domaine (graphique 4), dès la fin des années 1480, la production juridique baisse durablement, alors qu'elle avait eu son heure de gloire dans les années 1484-1490 ; cette chute est compensée par une hausse de la production littéraire. On observe également une tendance à la hausse de la production dans des formats plus petits (graphique 2).

Dans les années 1498-1499 il semble que le contenu des publications continue de changer de façon importante, ce qui est particulièrement flagrant pour le choix des auteurs et des domaines textuels, avec une nette baisse des auteurs médiévaux. La production en langue vulgaire, tous domaines confondus, connaît une hausse dans les deux dernières années, ce qui est à mettre en relation, inversement, avec une baisse de la production liturgique et paraliturgique, médicale et théologique, et surtout une hausse importante de la production littéraire italienne. Cependant, les secteurs en baisse ne le sont pas en valeur absolue : le nombre d'éditions se maintient à un niveau à peu près constant, ce qui dénote la permanence d'anciennes stratégies chez certains imprimeurs alors même que le modèle dominant est en train de changer.

Ces changements semblent correspondre à ce que l'on sait de la production des grands imprimeurs vénitiens au début du XVI $\mathrm{XI}^{\mathrm{e}}$ siècle : une production en italien, davantage tournée vers l'élite urbaine que vers l'université, des ouvrages d'assez large diffusion plutôt que des sommes juridiques ou théologiques qui avaient occupé les imprimeurs des années 1480. La comparaison entre les deux siècles est cependant risquée. Des bases de données existent pour le XVI eiècle, en particulier la base Edit16 qui a été intégrée récemment à la base de $1^{\prime} U S T C^{15}$. Une première recherche

\footnotetext{
${ }^{14}$ Les textes non commentés représentaient la quasi-totalité des éditions de textes dits « littéraires » avant 1475. Mais dans les années 1480, on passe à une proportion de 65-35\% entre les textes non commentés et les textes accompagnés de leur commentaire. La même évolution est visible pour les textes philosophiques: les éditions non commentées deviennent même périodiquement minoritaires à partir du début des années 1480 .

${ }^{15}$ La base Edit16, réalisée par l'ICCU (Istituto Centrale per il Catalogo Unico delle biblioteche italiane e per le informazioni bibliografiche) est consultable en ligne :
} 
à partir de cette base semble montrer que la progression de la production en italien continue bien par la suite. Cependant, Edit16 est affecté par un biais concernant les éditions italiennes, ce qui peut fausser l'analyse à la marge. Surtout, les catégories utilisées pour l'analyse sont très différentes de celle de la base d'Ezio Ornato et Claire Priol, ce qui rend les comparaisons très compliquées. Il n'empêche que l'évolution de certaines dynasties éditoriales vénitiennes va dans ce sens : il n'est pas rare d'observer que la production du fondateur de la dynastie au XV $\mathrm{VV}^{\mathrm{e}}$ siècle produisait surtout pour l'université, et en latin, alors que ses successeurs se tournent vers une littérature plus populaire $^{16}$.

Comment interpréter ces changements si l'on ramène l'analyse à l'échelle de l'atelier typographique ? Les presses vénitiennes au début de la période témoignent d'une grande capacité d'investissement, due en particulier aux capitaux allemands investis dans les compagnies de Nicolas Jenson et de Johann de Cologne. Ces deux grandes compagnies ont su élaborer un modèle éditorial répondant à la demande locale et internationale ${ }^{17}$. Ce modèle, une fois éprouvé, reste en vigueur même après leur disparition en 1480. Les années 1480, et même le début des années 1490 , ne sont que des variations sur une stratégie éditoriale qui a fait ses preuves. Mais celle-ci change à la fin des années 1490, à une époque où

www.edit16.iccu.sbn.it. Les recherches à l'échelle européenne sont également facilitées par le catalogue de l'USTC : www.ustc.ac.uk.

${ }^{16}$ C'est le cas notamment pour Melchior Sessa, actif de 1505 à 1555, et de son père, Giovanni Battista Sessa : F. Ascarelli et M. Menato, La Tipografia del '500 in Italia, Florence, 1989, p. 327. C'est également le cas de Bernardin Stagnino et de son parent Gabriele Giolito, étudié notamment par A. NuOvo dans « Da Trino a Venezia a Lione. Le imprese librarie dei mercanti trinesi », intervention au colloque Trino e l'arte tipografica nel XVI secolo, 13-14 avril 2014, disponible en ligne (www.academia.edu/8915555/Da_Trino_a_Venezia_a_Lione._Le_imprese_librarie_dei_merc anti_trinesi) ; et dans A. NuOvo et C. Coppens, I Giolito e la stampa nell'Italia del XVI secolo, Genève, 2005. Il n'empêche que certaines maisons d'édition continuent à être renommées pour leurs éditions érudites; ainsi, les œuvres en hébreu imprimées par Daniel Bomberg ou encore l'imprimerie des héritiers d'Alde, relativement réfractaire à la littérature en italien, comme le montre l'affaire de la publication du Courtisan de Castiglione: A. QUONDAM, «Questo povero Cortegiano ». Castiglione, il Libro, la Storia, Rome, 2000.

${ }^{17}$ Sur les débuts de l'imprimerie vénitienne, on peut se référer à P. BRAUNSTEIN, «Les Allemands et la naissance de l'imprimerie vénitienne », dans Revue d'études italiennes, vol. 27-4, 1981, p. 381-389, ainsi qu'à M. ZORZI, «Stampatori tedeschi a Venezia », dans Venezia e la Germania : arte, politica, commercio, due civiltà a confronto, Milan, 1986, p. 115-133. 
justement on observe une hausse des faillites, ou plus précisément une hausse du nombre d'imprimeurs qui ne demeurent actifs qu'un an ou deux ${ }^{18}$. Il devient de plus en plus difficile de s'établir à Venise et de maintenir son activité sur le long terme, signe que le modèle précédent a atteint ses limites. Les stratégies éditoriales doivent évoluer face à une demande sans doute plus diversifiée qu'aux débuts de l'imprimerie et un marché universitaire saturé : d'où un changement de paradigme, mais qu'on ne pourrait réellement observer qu'au début du XVI ${ }^{\mathrm{e}}$ siècle. La fin des années 1490 semble donc être un moment de transition vers un modèle différent, mais plus difficilement observable.

\section{VENISE DANS L'EUROPE DE L'INCUNABLE}

Les évolutions que nous avons retracées ici à grands traits sont loin d'être une exception en Europe avant 1500. Toutes les villes d'imprimerie connaissent une période de flottement dans les années qui suivent immédiatement l'introduction de la nouvelle technique. Pour ne prendre qu'un seul exemple, la production parisienne change beaucoup d'orientation entre les premières années et le début des années 1480. Dans les premiers temps, les presses parisiennes ont une volonté claire de se spécialiser dans la littérature puis la théologie, en lien avec les humanistes et l'université qui ont fait installer la première imprimerie dans la ville. Mais certains imprimeurs tentent également quelques éditions davantage tournées vers le droit ou les arts libéraux. À partir de 1483, on observe un repli sur la littérature morale et religieuse, avec un poids également important de la liturgie, de la théologie et des arts libéraux. Dominique Coq a étudié ces changements de tendances en soulignant l'importance du cercle humaniste dans les premières années et l'arrivée à partir de 1475 de nouveaux imprimeurs indépendants de l'université ; ceux-ci «élargissent leur clientèle en diversifiant leurs produits à destination d'un public de lecteurs traditionnels, ecclésiastiques et universitaires ... La flambée humaniste des débuts s'éteint progressivement » 19 .

${ }^{18}$ C. KIKUCHI, «Des Vagabonds des lettres ? Les typographes à Venise aux temps des débuts de l'imprimerie », journée d'études de Questes, 14-15 juin 2013, Précarité, instabilité, fragilité au Moyen Âge, à paraître aux Presses de l'université Paris-Sorbonne.

${ }^{19} \mathrm{D}$. CoQ, «Les politiques éditoriales des premiers imprimeurs parisiens et lyonnais (1470-1485) », dans Legenda aurea. Sept siècles de diffusion, Actes du colloque international, Montréal/Paris, 1986, p. 171-181. 
Par ailleurs, sur l'ensemble de la période, les choix éditoriaux de Venise semblent globalement cohérents par rapport aux choix des imprimeurs de l'espace italophone, tant du point de vue des langues publiées que des formats ou des contenus. Par opposition à ce «modèle italien », se dessine un «modèle allemand » qui se distingue par un poids important accordé à la production sacrée - $52 \%$ de la production allemande alors qu'elle ne représente que $36 \%$ de la production italienne hors Venise (tableau 2). La littérature qui y est produite est une littérature en latin traitant de sujets religieux ; la théologie y occupe également une place plus importante que dans l'espace italien; la production juridique y est bien moins développée (tableau 3). Ce tableau général est cependant très schématique et masque d'importantes évolutions chronologiques dans ces régions, l'Allemagne produisant de nombreux textes juridiques de base au début de la période.

Tableau 2 : Répartition de la production incunable vénitienne, italienne et allemande selon le caractère profane ou sacré de la publication

\begin{tabular}{|l|c|c|c|}
\hline & Venise & Italie & Allemagne \\
\hline Profane & $65,2 \%$ & $64,1 \%$ & $47,8 \%$ \\
\hline Sacré & $33,3 \%$ & $35,9 \%$ & $52,2 \%$ \\
\hline
\end{tabular}

Tableau 3 : Répartition de la production incunable vénitienne, italienne et allemande par domaine

\begin{tabular}{|l|c|c|c|}
\hline & Venise & Italie & Allemagne \\
\hline arts libéraux & $9,7 \%$ & $8,8 \%$ & $12,4 \%$ \\
\hline droit & $18,8 \%$ & $18,1 \%$ & $5,0 \%$ \\
\hline histoire & $6,0 \%$ & $5,7 \%$ & $4,2 \%$ \\
\hline littérature & $28,3 \%$ & $33,7 \%$ & $27,9 \%$ \\
\hline liturgie et Bible & $9,7 \%$ & $5,4 \%$ & $10,5 \%$ \\
\hline médécine & $4,3 \%$ & $4,4 \%$ & $2,8 \%$ \\
\hline philosophie & $6,8 \%$ & $4,5 \%$ & $4,0 \%$ \\
\hline sciences & $4,3 \%$ & $3,6 \%$ & $1,8 \%$ \\
\hline théologie & $7,0 \%$ & $8,1 \%$ & $12,1 \%$ \\
\hline vie politique et administrative & $1,2 \%$ & $2,5 \%$ & $9,8 \%$ \\
\hline vie quotidienne & $2,9 \%$ & $4,1 \%$ & $8,0 \%$ \\
\hline autres & $0,9 \%$ & $1,1 \%$ & $1,0 \%$ \\
\hline
\end{tabular}

Si l'on compare Venise avec les villes d'imprimerie les plus proches en Italie du Nord, on constate que, sans pour autant se démarquer par des choix 
éditoriaux radicalement différents, la ville réussit à étouffer la concurrence des autres centres qui ne réussissent jamais à décoller véritablement. La production totale du duché de Ferrare et celle du duché de Milan restent à un faible niveau alors que celle de Venise décolle tout au long du Xve siècle. Dans le territoire sous domination directe de Venise, la Terre Ferme, la production reste également faible, plus soumise aux fluctuations du marché et aux mouvements des imprimeurs (graphique 6)20. Dans cet espace, on ne trouve que trois villes ayant une production continue : Brescia, Padoue et Vicence ; mais la production de Padoue, trop proche de Venise, baisse de façon importante dès 1479 .

Venise n'impose pas de monopole sur son territoire, elle n'empêche pas l'ouverture de presses en Terre Ferme ; cependant, les privilèges qu'elle accorde à partir des années 1480 sont dans leur écrasante majorité destinés à des imprimeurs actifs à Venise et leur assurent le monopole de la production et de la commercialisation de certaines ouvres sur tout le domaine. Cela a sans doute contribué à l'asphyxie des imprimeries de Terre Ferme sur la fin de la période. Plus largement, les imprimeries locales - celles de Padoue en particulier, mais pas uniquement - deviennent les relais des imprimeurs vénitiens qui y installent leurs succursales et concluent des contrats avec les imprimeurs locaux. Elles sont aussi des viviers pour les presses vénitiennes : beaucoup d'imprimeurs actifs à Venise proviennent des villes d'Italie du Nord, sous domination vénitienne ou non. On compte en particulier de nombreux imprimeurs et éditeurs originaires du Montferrat, du Milanais, de Brescia ou encore de Florence. Si certains d'entre eux sont mobiles et s'installent alternativement dans différentes villes italiennes, d'autres fondent leur atelier à Venise et le font fonctionner sur le long terme. Les citoyens de Terre Ferme n'hésitent pas à faire valoir leur lien légal à Venise pour solliciter des privilèges 21 . En somme, l'imprimerie vénitienne a réussi jusqu'à la fin du xve siècle à drainer une bonne partie des forces vives de l'imprimerie naissante en Italie du Nord à son profit.

${ }^{20}$ Dans le graphique 6, nous comparons la production de Venise avec celle de territoires comprenant plusieurs villes d'imprimerie. L'écart ne signale que davantage le poids de Venise dans son environnement régional.

21 Ainsi, Piero de Benali dans une demande de privilège de 1494: «Presbyter Petrus de Benaliis fidelis civis noster Bergomensis et habitator Venetiarum»: Archivio di Stato di Venezia (ASV), Collegio, Notatorio, reg. 14-22, image 196 (fond numérisé) ; ou encore Paganino de Paganini la même année : «el fidelissimo servitor et subdito de vostra signoria Paganino de Paganini, vostro citadino di Bressa et impressor diligentissimo de libri », ASV, Collegio, Notatorio, reg. 14-22, image 228. 
Cette comparaison rapide avec certains des grands centres du livre imprimé européen à la fin $d u X V^{\mathrm{e}}$ siècle permet de mieux situer Venise dans le contexte de la production européenne. La cité n'est pas isolée dans ses évolutions ni dans sa production. L'introduction de l'imprimerie a suscité d'importantes hésitations sur le type d'œuvres susceptible de rencontrer le public: pour aller au-delà du public traditionnel des manuscrits, les imprimeurs ont dû partout tâtonner pour aboutir à des choix et des stratégies éditoriales cohérentes. Les grandes tendances de l'imprimerie vénitienne semblent en adéquation avec les stratégies des imprimeurs italiens dans leur ensemble. Cette réussite a condamné la progression des imprimeries voisines, et a été permise, en même temps, par l'arrivée de sang neuf, venu du territoire sous domination vénitienne et au-delà, du Milanais ou du Montferrat par exemple. Ce processus cumulatif a joué en faveur d'un développement rapide et continu, sans pour autant que Venise développe une stratégie éditoriale particulièrement originale en comparaison des autres villes italiennes.

\section{UN MODELE EDITORIAL VENITIEN?}

Mais alors, y a-t-il une spécificité des stratégies éditoriales vénitiennes ? Ou bien celles-ci ne sont-elles que le reflet à une échelle plus fine des stratégies à l'œuvre dans toute l'Italie à la même époque ?

Intéressons-nous à un cas précis : la publication d'auteurs contemporains de l'édition. Nous avons pour cela tenu compte de toutes les éditions dont l'auteur est décédé l'année de la publication ou ultérieurement (graphique 7). Contrairement à ce à quoi on pourrait s'attendre au vu de ce qui se produit ailleurs, il n'y a pas de tendance nette à l'augmentation de la part d'auteurs vivants ou contemporains dans la production vénitienne, même si leur nombre augmente avec le temps. La proportion d'auteurs vivants est globalement faible, oscillant entre 10 et $20 \%$. Cette situation est assez surprenante compte tenu de la politique menée par d'autres villes d'imprimerie italiennes (graphique 8). Ainsi Florence publie une forte proportion d'auteurs vivants, qui atteint même près de la moitié de sa production dans les quatre dernières années du siècle (93 éditions sur 188). Cela dit, la production florentine est bien plus faible : on ne compte que 11 éditions durant les huit premières années d'activité et ce n'est que dans les quatre années entre 1489 et 1492 que Florence dépasse la centaine d'éditions produites. Dans le cas de Ferrare, la production est encore plus faible - entre 6 et 28 éditions par tranche de quatre ans -, mais les auteurs vivants en représentent une part importante. De la même manière, en Italie 
en dehors de Venise, les auteurs vivants représentent $31,9 \%$ de la production totale, avec une tendance nette à l'augmentation sur la fin de la période. Or, entre 1469 et 1500 , ils ne représentent que $15,5 \%$ de la production vénitienne sans véritable tendance à l'augmentation au cours de la période (tableau 3). Le décalage ici est particulièrement flagrant entre Venise et les autres villes d'imprimerie italiennes.

Tableau 3 : Part des auteurs vivants dans la production incunable vénitienne et italienne

\begin{tabular}{|l|r|r|r|}
\hline & $1469-1476$ & $1493-1500$ & Ensemble \\
\hline Venise & $16,6 \%$ & $17,2 \%$ & $\mathbf{1 5 , 4 \%}$ \\
\hline Espace italophone & $19,9 \%$ & $29,8 \%$ & $\mathbf{2 1 , 9 \%}$ \\
\hline
\end{tabular}

À titre de comparaison, aux Pays-Bas, selon Chiara Ruzzier et Xavier Hermand, on compte $23 \%$ d'auteurs publiés de leur vivant dans la production totale, avec un accroissement de cette proportion sur la fin de la période22.

Pourquoi cette spécificité vénitienne ? Elle est à mettre en rapport avec le nombre moyen de rééditions par ouvrage : pour l'ensemble de la période et de la production, le nombre moyen d'édition du même ouvrage s'élève à 2,21. Le même indicateur aux Pays-Bas était compris entre 1,32 et 1,99, selon la langue et la période considérée, chiffre déjà jugé comme élevé-23. Le nombre de rééditions est donc particulièrement important à Venise, sur une production elle-même supérieure en nombre à celle de toute autre ville européenne.

Chiara Ruzzier et Xavier Hermand interprètent le nombre de rééditions et la publication d'auteurs vivants comme des indicateurs de l'originalité des politiques éditoriales et de la propension des imprimeurs à la prise de risque. Dans le cas de Venise, il est clair que la ville ne connaît pas une politique aussi proche de l'activité intellectuelle contemporaine qu'on aurait pu le croire : elle en est même moins proche que les autres villes italiennes. Mais est-ce vraiment surprenant? On peut déceler dans le fort pourcentage d'auteurs vivants publiés à Ferrare ou à Bologne une spécificité des plus petits centres d'imprimerie qui sont également des centres intellectuels et culturels. L'exemple de Ferrare est particulièrement frappant: sur 106 éditions incunables, 35 sont des publications d'auteurs vivants; on compte alors 23 auteurs différents dont au moins 16 ont un lien direct avec la ville,

${ }^{22}$ C. RuZZier, X. Hermand, E. Ornato, Les Stratégies éditoriales... (op. cit.), p. 74. ${ }^{23}$ Ibid., p. 75. 
que ce soit par le biais de la cour ducale ou par le biais de l'université. Sur une faible production, il est clair ici qu'il y a une volonté de promouvoir les auteurs locaux, actifs dans la ville ou qui l'ont été dans un passé proche. Mais il s'agit d'une prime à la proximité, qui traduit aussi le fait que l'imprimerie de Ferrare souffre de la concurrence des autres grands centres italiens : son marché peine à dépasser l'échelle locale et régionale. Les auteurs publiés sont pour l'essentiel des auteurs à portée restreinte, si l'on excepte quelques grands noms tels que Savonarole ou Battista Guarini.

Si l'on compare la situation de Ferrare et celle de Venise, il est clair que nous ne sommes pas sur la même échelle et donc pas dans la même optique éditoriale, ce qui explique que le nombre d'auteurs publiés de leur vivant soit plus faible à Venise. Cela expliquerait également la ressemblance de la situation vénitienne avec celle d'autres grandes villes d'imprimerie, telle que Cologne, qui publie également peu d'auteurs vivants. La ville allemande connait elle aussi des périodes de faible production contemporaine, même si celle-ci peut occasionnellement s'élever à un tiers de sa production totale. Plutôt que d'imprimer l'oeuvre de lettrés locaux, les imprimeurs des grands centres d'imprimerie, et particulièrement de Venise, préfèrent donc rééditer des textes qui ont déjà fait leurs preuves, afin de toucher un public européen le plus large possible. Quand ils publient des auteurs vivants, ce sont des noms dont la notoriété dépasse largement l'échelon local: on trouve des papes, des savants allemands, espagnols, qui témoignent du large public et des aires géographiques éloignées que les imprimeurs et éditeurs cherchent à atteindre.

L'exemple de la publication d'auteurs contemporains permet de mieux comprendre ce que pouvait être le modèle éditorial vénitien en construction durant la période de l'incunable. Les changements de fond ou de forme que connait la production vénitienne répondent tous au même impératif : celui de toucher des marchés éloignés et de rendre rentable l'investissement grâce à des tirages importants. D'où une préférence pour les ouvrages universitaires, les rééditions, et une certaine frilosité envers les auteurs vivants. Les imprimeurs cherchent à se stabiliser dans un environnement hautement concurrentiel en publiant des ouvrages de droit et de littérature, qui touchent le public universitaire et lettré européen, sans pour autant négliger complètement les ouvrages liturgiques et de piété commune, dont la diffusion se fait également facilement24. Mais cet impératif conduit parfois à des choix qui peuvent sembler contradictoires avec une volonté de stabilité

${ }^{24}$ Notons d'ailleurs qu'un certain nombre d'imprimeurs peuvent produire des livres liturgiques pour des diocèses éloignés, comme le montrent les éditions de bréviaires pour Ezstergom ( $\mathrm{N}^{\circ}$ ISTC : ib01183050), Saragosse ( $\mathrm{N}^{\circ}$ ISTC : ib01150450), Salzbourg ( ${ }^{\circ}$ ISTC $:$ ib01178000) ou encore Salisbury ( $N^{\circ}$ ISTC : ib01178330). 
et de prudence. Certains imprimeurs comme Andrea Torresani investissent dans des caractères « exotiques », comme le glagolitique, afin de toucher le marché catholique dalmate et croate25; son associé Alde Manuce réussit à le convaincre qu'une fonte de caractères grecs peut être un investissement rentable 26 ; d'autres, comme Erhard Ratdolt, publient des auteurs allemands pour toucher le marché germanique, de première importance pour Venise. Ces choix éditoriaux ne sont pas uniquement des cas-limites et exceptionnels : la volonté de conquérir de nouveaux publics permet de résoudre le paradoxe apparent de la production vénitienne, extrêmement originale par certains aspects, et au contraire très conservatrice par d'autres. Dans le cas de l'investissement lourd que représente la taille de nouveaux caractères pour un alphabet non latin comme dans celui d'un nombre important de rééditions d'auteurs bien établis, il s'agit de diffuser les ouvrages dans toute l'Europe, un objectif que les imprimeurs vénitiens ont donc très rapidement intégré et pris en compte.

Les stratégies individuelles qui sont ensuite mises en place dépendent très largement de la capacité d'investissement de chaque imprimeur, comme nous avons commencé à le voir avec les éditions d'auteurs vivants. Une étude plus fine et systématique reste à faire à l'échelle des ateliers vénitiens. Concernant les capacités d'investissement, cette étude se heurte à un problème de taille: de nombreuses d'éditions sont co-financées par un imprimeur et un bailleur de fonds, qui peut être mentionné ou non dans le colophon. Pour ne prendre qu'un seul exemple célèbre, le patricien Pierfrancesco Barbarigo est un investisseur indispensable à l'imprimerie aldine, mais n'apparaît jamais dans les imprimés : il a fallu attendre la découverte des papiers réglant sa succession pour prendre la mesure de son importance financière dans l'imprimerie dirigée par Alde Manuce et son associé Andrea Torresani27. Cet exemple montre qu'il faut interpréter avec prudence les relations entre imprimeurs et l'origine de leurs fonds, dont dépendent largement leurs stratégies éditoriales.

Cependant, l'étude de quelques cas emblématiques nous laisse bien voir que la relative diversité des éditions vénitiennes cache en réalité une spécialisation interne forte. Certains imprimeurs concentrent la moitié ou plus de leur production dans le droit, en particulier Bernardin Stagnino, Baptista de Torti, Andrea Torresani (seul) et la compagnie de Johann de Colonia et Johann Manthen. D'autres se spécialisent, au contraire, dans la

25 S. PELUSI, «La Stampa in caratteri glagolitici e cirillici », dans Armeni, ebrei, greci stampatori a Venezia, Scilla Abbiati (dir.), Venise, 1989, p. 101-114.

${ }^{26}$ M. Lowry, Le Monde d'Alde Manuce, op. cit., chapitre «Barbarigo, Torresani et Manuce », p. 81-117.

${ }^{27}$ Ibid., p. 91-94. 
littérature et les arts libéraux, comme Simon Bevilaqua ou encore Cristoforo de Pensis. Quelques individus privilégient des productions plus étranges dans le paysage vénitien, tel Johann Hamman dont la moitié de la production consiste en ouvrages liturgiques, ou encore Boneto Locatelli qui poursuit un programme éditorial éclectique entre la médecine, la philosophie et la théologie (tableau 5). La diversité des politiques éditoriales de ces grands ateliers permet de nuancer le tableau que nous avons dressé jusqu'ici, même si ces quelques exemples demandent à être davantage approfondis dans des études ultérieures. Cependant, il est clair que, suivant leurs capacités de production et d'investissement, selon leurs réseaux commerciaux, les imprimeurs vénitiens ne suivent pas tous la même stratégie éditoriale, même si celles-ci s'inscrivent dans un schéma général vénitien de conquête de nouveaux marchés européens.

TABLEAU 5. - Part des différents domaines dans la production de quelques imprimeurs vénitiens.

\begin{tabular}{|l|c|c|c|c|c|c|c|c|}
\hline & $\begin{array}{c}\text { Total } \\
\text { des } \\
\text { éditions }\end{array}$ & $\begin{array}{c}\text { arts } \\
\text { libéraux }\end{array}$ & droit & littérature & liturgie & médecine & $\begin{array}{c}\text { philo- } \\
\text { sophie }\end{array}$ & théologie \\
\hline $\begin{array}{l}\text { Andreas } \\
\text { Torresanus }\end{array}$ & $\mathbf{1 3 1}$ & $2,30 \%$ & $51,10 \%$ & $5,30 \%$ & $29,80 \%$ & $0,80 \%$ & $3,10 \%$ & $3,10 \%$ \\
\hline $\begin{array}{l}\text { Johannes de } \\
\text { Colonia }\end{array}$ & $\mathbf{8 2}$ & $3,70 \%$ & $46,30 \%$ & $17,10 \%$ & $1,20 \%$ & $2,40 \%$ & $4,90 \%$ & $15,90 \%$ \\
\hline $\begin{array}{l}\text { Baptista de } \\
\text { Tortis }\end{array}$ & $\mathbf{1 6 8}$ & $5,40 \%$ & $65,50 \%$ & $17,90 \%$ & $1,80 \%$ & $3,60 \%$ & $4,80 \%$ & $0 \%$ \\
\hline $\begin{array}{l}\text { Bernardinus } \\
\text { Stagninus }\end{array}$ & $\mathbf{8 9}$ & $0 \%$ & $76,40 \%$ & $3,40 \%$ & $4,50 \%$ & $5,60 \%$ & $3,40 \%$ & $2,20 \%$ \\
\hline $\begin{array}{l}\text { Simon } \\
\text { Bevilaqua }\end{array}$ & $\mathbf{7 5}$ & $8,00 \%$ & $4,00 \%$ & $41,30 \%$ & $5,30 \%$ & $4,00 \%$ & $14,70 \%$ & $6,70 \%$ \\
\hline $\begin{array}{l}\text { Christoforus } \\
\text { de Pensis }\end{array}$ & $\mathbf{7 3}$ & $21,90 \%$ & $0 \%$ & $47,90 \%$ & $2,70 \%$ & $5,50 \%$ & $1,40 \%$ & $2,70 \%$ \\
\hline $\begin{array}{l}\text { Johannes } \\
\text { Hamman }\end{array}$ & $\mathbf{8 5}$ & $3,50 \%$ & $7,10 \%$ & $4,70 \%$ & $55,30 \%$ & $4,70 \%$ & $4,70 \%$ & $2,40 \%$ \\
\hline $\begin{array}{l}\text { Bonetus } \\
\text { Locatellus }\end{array}$ & $\mathbf{1 5 3}$ & $7,20 \%$ & $1,30 \%$ & $13,70 \%$ & $2,00 \%$ & $21,60 \%$ & $26,80 \%$ & $13,70 \%$ \\
\hline
\end{tabular}

\section{CONCLUSION}

L'étude de la production et des stratégies éditoriales des imprimeurs nous permet de mieux comprendre la manière dont l'imprimerie s'est installée et stabilisée dans les villes où elle s'est implantée. Dans le cas de Venise, l'étude est d'autant plus intéressante qu'il s'agit du premier pôle de production incunable en Europe, mais c'est précisément ce qui rend une étude fine d'autant plus complexe. Beaucoup reste à faire, en particulier une analyse plus fine des stratégies éditoriales par ateliers, notamment en 
fonction des liens économiques et sociaux qui peuvent exister entre eux. Nous avons tenté ici de dresser les grandes évolutions de la production vénitienne entre 1469 et 1500 , en les replaçant dans leur contexte régional et européen d'une part, et dans leur contexte économique et social d'autre part. Il s'agissait également de comprendre la spécificité de Venise, au-delà du point de vue purement quantitatif, d'expliquer certaines des raisons du succès de son industrie, sans se laisser abuser par les imprimeurs les plus célèbres, qui donnent une image biaisée de ce qu'était réellement la production du livre dans la ville.

Venise a d'emblée visé le marché lettré européen, au-delà du marché universitaire padouan ou des humanistes locaux, même si le marché le plus proche lui a sans doute servi de tremplin. Sa production est relativement diversifiée, avec cependant une nette spécialisation dans le droit et la littérature. Les modifications de la fin de la période ne font que témoigner de l'adaptabilité de ces entreprises typographiques, voire de l'arrivée aux commandes d'une nouvelle génération, qui cherche à atteindre un public urbain, qui n'est plus uniquement lettré ou universitaire. La prudence et l'audace ont chacune leur part dans les stratégies des ateliers de la ville, mais toujours en vue de conquérir de nouveaux marchés, par opposition à des centres d'imprimerie qui apparaissent plus en phase avec la production intellectuelle contemporaine, mais qui en réalité sont tributaires d'un marché local et d'un rayonnement commercial réduit. Ces évolutions témoignent enfin de l'organisation du milieu du livre vénitien, dominé jusqu'aux années 1480 par deux grandes compagnies, et beaucoup plus morcelé par la suite. Cela a des conséquences sur la capacité d'investissement des imprimeurs, leur degré de prudence et donc sur les stratégies qu'ils sont susceptibles d'adopter.

Graphique 1 : Évolution de la production profane et sacrée à Venise entre 1469 et 1500

Graphique 2 : Évolution de la production selon le format à Venise entre 1469 et 1500

Graphique 3 : Évolution de la production en italien et en latin à Venise entre 1469 et 1500

Graphique 4 : Évolution de la production vénitienne incunable dans les principaux domaines de publication

Graphique 5 : Part des auteurs selon leur date de mort dans la production vénitienne incunable

Graphique 6: La production incunable vénitienne comparée à la production italienne voisine (en nombre d'éditions) 
Graphique 7 : Part d'auteurs vivants dans la production vénitienne incunable

Graphique 8 : Part des auteurs vivants dans la production incunable de différentes villes (moyenne mobile de deux tranches triennales)

(Eliminer les légendes correspondantes des figures) 